\title{
Mapping Cool Gas in the ISM with ROSAT
}

\author{
I.B. Hutchinson, R.S. Warwick, and R. Willingale \\ Department of Physics and Astronomy, University of Leicester, University Road, \\ Leicester, LE1 7RH, UK
}

\begin{abstract}
We have investigated the distribution of cool gas within the local interstellar medium $(<150 \mathrm{pc})$ using data derived from both the ROSAT X-ray and EUV all-sky surveys. The EUV/X-ray spectra for a sample of 89 white dwarf stars and 200 active late-type stars have been analysed to give an estimate of the gas column density along each object's line of sight. In order to extend the sky coverage we have also included in the analysis 190 late-type stars detected only in the X-ray band. The derived $N_{H I}$ measurements confirm the size of the local low density bubble as typically 50-100 pc and also show that in the general direction of Loop I there is a substantial excess of absorption at a range of distances from 10-150 pc.
\end{abstract}

\section{Introduction}

Over the last twenty years a detailed picture of the interstellar medium in the vicinity of the Sun has been built-up on the basis of the optical and UV absorption features observed in the spectra of nearby stars and from studies of the soft X-ray background (see Frisch 1997). In particular, there now exists a large database of hydrogen column density measurements, $N_{H I}$, which can be used to trace the distribution of cool gas at distances from a few out to many hundreds of parsecs (Fruscione et al. 1994). One feature of the local cool gas distribution which has been highlighted by the recent EUV all-sky surveys carried out by the ROSAT WFC and EUVE (Pounds et al. 1993; Pye et al. 1995; Bowyer et al. 1994 ; Barber et al. (1996) ) is an apparent nearby "wall" of absorption observed in the general direction of the Galactic centre as revealed by the relative paucity of EUV source detections in this region (e.g. Warwick et al. 1993). Here we use a combination of EUV and X-ray data derived from the ROSAT all-sky survey to investigate the distribution of absorbing gas in the direction of this wall.

\section{Data Analysis}

The first part of our study is based on the EUV sources listed in the ROSAT WFC 2RE catalogue (Pye et al. 1995). The properties of a total of 89 white dwarf stars drawn from this survey have been discussed recently by Marsh et al. (1997). These authors list $N_{H I}$ values for each source derived from fitting detailed atmospheric models to a combination of WFC S1 $(60-140 \AA)$ and 
S2 (112-200 $\AA$ ) filter measurements and ROSAT PSPC (5.2-100 $\AA)$ data. We also cross-correlated the $2 \mathrm{RE}$ catalogue with the public version of the ROSAT PSPC bright source catalogue (RBSC) and selected a sample of 200 late-type stars common to both lists. The data from the RBSC were available in the form of three hardness ratios and a broad band count-rate, which we combined with the S1 and S2 measurements to give effectively five energy channels. Spectral models for the emission from late-type stars typically include a column density and two thermal plasma components, requiring in total five spectral parameters. For our purpose we set the two temperatures and also the ratio of the normalisations of the thermal components to values typical of the data set as a whole and then performed a spectral fitting analysis to derive an estimate for $N_{H I}$ for each source. The sky distribution of the white dwarf stars and late-type stars which comprise the WFC sample is shown in Fig. 1.

In an attempt to obtain greater sky coverage and to increase the distance range sampled we have also extended our study to include all the sources in the RBSC catalogue which cross-correlated with known late-type stars contained in the Hipparcos Input Catalogue. For these sources we have only three effective energy channels, but again we were able to estimate the line-ofsight $N_{H I}$ using the spectral model as described above. Since the PSPC-only measurements are insensitive to $N_{H I}$ values less than $\sim 3 \times 10^{19} \mathrm{~cm}^{-2}$, for the present work we restrict our attention to stars at distances greater than 50 parsecs. The sky distribution of the PSPC-only sample of 190 late-type stars is shown in Fig.2.

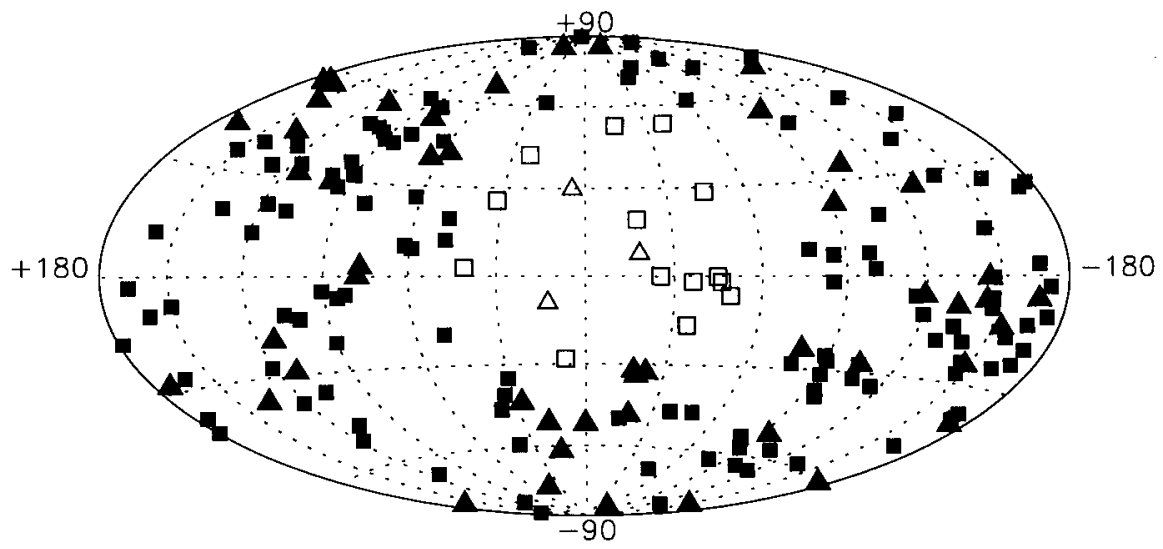

Fig. 1. The sky distribution in galactic coordinates of the white dwarf stars (triangles) and late-type stars (boxes) in the WFC sample. Stars inside the the nominal wall region are shown as open symbols. 


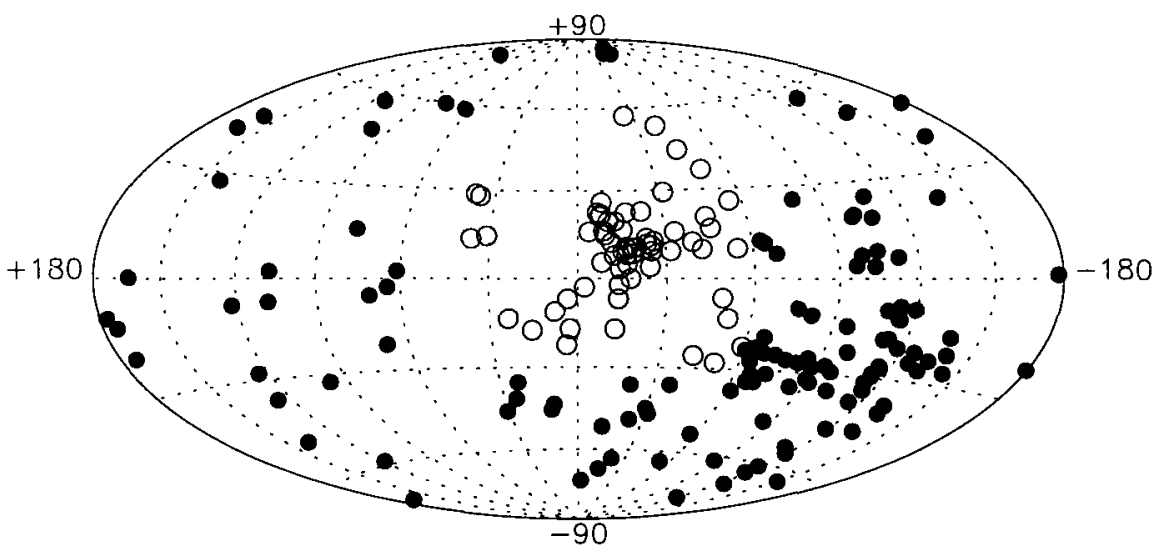

Fig. 2. The sky distribution in galactic coordinates of the late-type stars in the PSPC-only sample. Stars within the wall region are shown as open circles.

\section{The 'Wall' of Absorption Towards the Galactic Centre}

The sparse number of EUV source detections in the general direction of the Galactic centre has been noted in a number of studies. Based on a detailed analysis of the statistics pertaining to the ROSAT WFC survey, Warwick et al. (1993) concluded that the effective edge of the local bubble in this region is anomalously close to the Sun i.e. at $\sim 10 \mathrm{pc}$ as opposed to a more typical radius for the bubble of $80-100 \mathrm{pc}$ ). The presence of relatively nearby clouds in this part of the sky is evident from the optical/UV absorption studies of Frisch \& York (1983) and Paresce (1984). The region also coincides with the Loop I superbubble (Egger 1997). Here we define the wall region as that bounded by $45^{\circ}>l>300^{\circ}$ and $-30^{\circ}<b<60^{\circ}$.

Figure 3 shows the median value of $N_{H I}$ measured in various distance ranges for sources selected inside and outside of the wall region. (We use median values since each individual $N_{H I}$ measurement is subject to fairly large errors, a more detailed analysis of which is outside the scope of the present paper.) The trend in the data in Fig. 3 is clear, namely that the column density measurements show a significant up-turn at distances of 50$100 \mathrm{pc}$ in many directions. The wall region is characterised by excess column density compared to the all-sky norm but the data do not identify any specific cloud structure in this direction.

\section{Discussion}

The combination of EUV and X-ray data from the ROSAT all-sky survey for nearby stars provides us with a method of studying the distribution of 


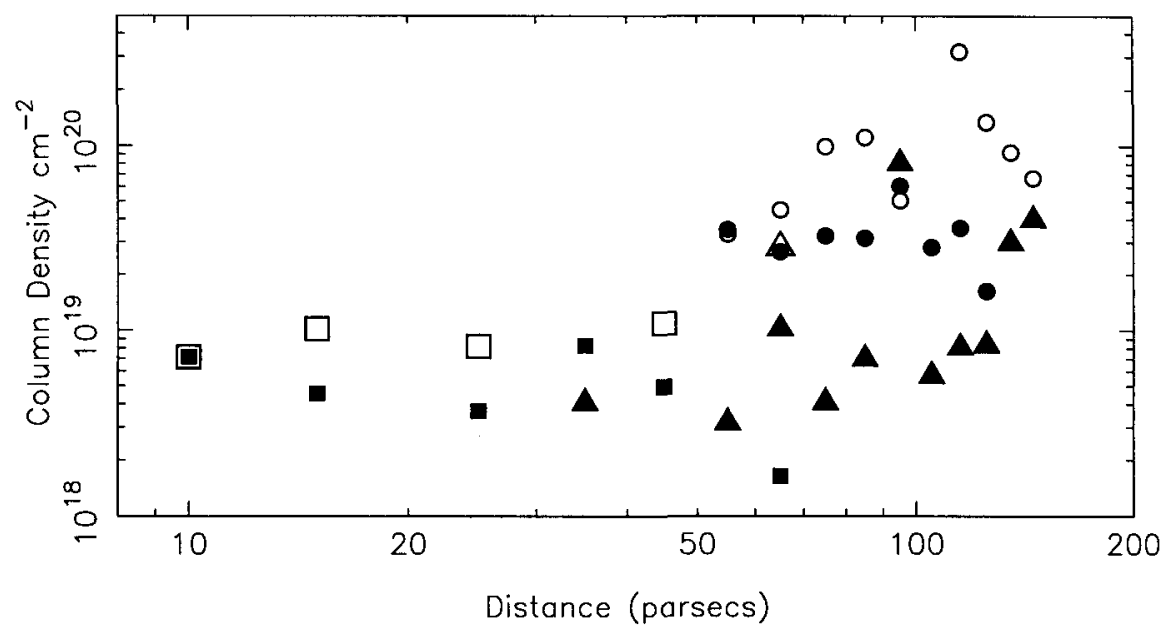

Fig. 3. The median value of the column density, $N_{H I}$, measured for the various source samples in different distance ranges. The late-type and white dwarfs stars from the WFC survey are shown as squares and triangles respectively, whereas the late-type stars drawn only from the RBSC are shown as circles. The open (filled) symbols correspond to those stars located within (outside) the region of the wall.

cool gas in the vicinity of the Sun. This approach can supplement the information available from the optical and UV absorption line studies. Here we have presented a very preliminary analysis emphasising a selected area of the sky (the general region of the Galactic centre) but the data are relevant to the global distribution of absorbing material over distance scales from 10 to several hundred parsecs. Future work will make use of the refined stellar distances and parameters available from the Hipparcos mission.

\section{References}

Bowyer, S., et al. (1994): ApJS, 93, 569

Bowyer, S., et al. (1996): ApJS, 102, 129

Egger, R., (1997): these proceedings

Frisch, P., York, D., (1983): ApJ, 271, L59

Frisch, P., (1997): these proceedings

Fruscione, A., et al. (1994): ApJS, 94, 127

Marsh, M., et al. (1997): MNRAS, 286, 369

Paresce, F., (1984): AJ, 89, 1022

Pounds, K., et al. (1993): MNRAS, 260, 77

Pye, J., et al. (1995): MNRAS, 274, 1165

Warwick R., Barber C., Hodgkin S., Pye J. (1993): MNRAS, 262, 289 